\title{
Erratum: On Regularity Lemmas and their Algorithmic Applications
}

\author{
JACOB FOX ${ }^{1}$, LÁSZLÓ MIKLÓS LOVÁSZ ${ }^{2}$ and YUFEI ZHAO ${ }^{3}$ \\ ${ }^{1}$ Department of Mathematics, Stanford University, Stanford, CA 94305, USA \\ (e-mail: jacobfox@stanford.edu) \\ 2 Department of Mathematics, UCLA, Los Angeles, CA 90095, USA \\ (e-mail: lmlovasz@math.ucla.edu) \\ ${ }^{3}$ Department of Mathematics, MIT, Cambridge, MA 02139, USA \\ (e-mail: yufeiz@mit.edu)
}

Received 12 January 2018; revised 7 February 2018; first published online 22 March 2018

Our paper [3] contained two errors.

We retract Corollary 3.5. We claimed that it follows from a recent algorithmic version [1,2] of the Frieze-Kannan regularity lemma (see Theorems 3.2 and Theorem 3.3 in [3]). Unfortunately there is an error in our application of these algorithmic results. Although the algorithms in $[1,2]$ can test whether a partition is weakly regular, it is unclear how to apply them to an intermediate step in our proposed algorithm. Specifically, we would need an algorithm that, given

$$
G^{\prime}=d(G)+c_{1} K_{S_{1}, T_{1}}+\cdots+c_{l} K_{S_{l}, T_{l}},
$$

either (1) correctly states that $d_{\square}\left(G, G^{\prime}\right) \leqslant \varepsilon$, or (2) outputs sets $S$ and $T$ such that $\mid e_{G}(S, T)-$ $e_{G^{\prime}}(S, T) \mid>\varepsilon^{-O(1)}$. In particular, the fact that $G^{\prime}$ may not have bounded weights presents a challenge in applying results from [1,2].

A second error is in the proof of Theorem 1.4 in [3], which we also retract. We erred in applying a counting lemma [3, Lemma 4.1] to claim that if $G^{\prime}$, as above, approximates $G$ in cut norm, then $G$ and $G^{\prime}$ have similar $H$-densities. The counting lemma requires the edge-weights to be bounded by 1 , which is not necessarily true in this case.

We have been able to fix Corollary 3.5. In our new paper [4], we prove a strengthened version of the algorithm whose running time has improved dependence on $\varepsilon$ and $n$. Specifically, we prove the following theorem to replace Corollary 3.5. 
Theorem 1. There is a deterministic algorithm that, given $\varepsilon>0$ and an n-vertex graph $G$, outputs, in $\varepsilon^{-O(1)} n^{2}$ time, subsets $S_{1}, S_{2}, \ldots, S_{r}, T_{1}, T_{2}, \ldots, T_{r} \subseteq V(G)$ and

$$
c_{1}, c_{2}, \ldots, c_{r} \in\left\{-\frac{\varepsilon^{8}}{300}, \frac{\varepsilon^{8}}{300}\right\}
$$

for some $r=O\left(\varepsilon^{-16}\right)$, such that

$$
d_{\square}\left(G, d(G)+c_{1} K_{S_{1}, T_{1}}+\cdots+c_{r} K_{S_{r}, T_{r}}\right) \leqslant \varepsilon .
$$

We have been able to salvage the following weaker result to replace Theorem 1.4 in [3] (with an improved dependence on $n$ thanks to Theorem 1 above).

Theorem 2. There is a deterministic algorithm that, given $\varepsilon>0$, a graph $H$, and an $n$-vertex graph $G$, outputs, in $O\left(\varepsilon^{-O_{H}(1)} n^{2}\right)$ time, the number of copies of $H$ in $G$ up to an additive error of at most $\varepsilon n^{v(H)}$.

See [4] for proofs and discussion.

\section{References}

[1] Dellamonica, D., Kalyanasundaram, S., Martin, D., Rödl, V. and Shapira, A. (2012) A deterministic algorithm for the Frieze-Kannan regularity lemma. SIAM J. Discrete Math. 26 15-29.

[2] Dellamonica Jr, D., Kalyanasundaram, S., Martin, D. M., Rödl, V. and Shapira, A. (2015) An optimal algorithm for finding Frieze-Kannan regular partitions Combin. Probab. Comput. 24 407-437.

[3] Fox, J., Lovász, L. M. and Zhao, Y. (2017) On regularity lemmas and their algorithmic applications. Combin. Probab. Comput. https://doi.org/10.1017/S0963548317000049

[4] Fox, J., Lovász, L. M. and Zhao, Y. A fast new algorithm for weak graph regularity. arXiv:1801.05037 